\title{
Lateralized electrical brain activity reveals covert attention allocation during speaking
}

\author{
Joost Rommers ${ }^{\mathrm{a}, \mathrm{b}, *}$, Antje S. Meyer ${ }^{\mathrm{a}, \mathrm{c}}$, Peter Praamstra ${ }^{\mathrm{b}, \mathrm{c}}$ \\ a Max Planck Institute for Psycholinguistics, Nijmegen, The Netherlands \\ b Department of Neurology, Radboud University Nijmegen Medical Centre, Nijmegen, The Netherlands \\ ${ }^{\mathrm{c}}$ Donders Institute for Brain, Cognition, and Behaviour, Radboud University Nijmegen, Nijmegen, The Netherlands
}

\section{A R T I C L E I N F O}

\section{Keywords:}

Language production

Object naming

Covert attention

EEG

N2pc

\begin{abstract}
A B S T R A C T
Speakers usually begin to speak while only part of the utterance has been planned. Earlier work has shown that speech planning processes are reflected in speakers' eye movements as they describe visually presented objects. However, to-be-named objects can be processed to some extent before they have been fixated upon, presumably because attention can be allocated to objects covertly, without moving the eyes. The present study investigated whether EEG could track speakers' covert attention allocation as they produced short utterances to describe pairs of objects (e.g., "dog and chair"). The processing difficulty of each object was varied by presenting it in upright orientation (easy) or in upside down orientation (difficult). Background squares flickered at different frequencies in order to elicit steady-state visual evoked potentials (SSVEPs). The N2pc component, associated with the focusing of attention on an item, was detectable not only prior to speech onset, but also during speaking. The time course of the N2pc showed that attention shifted to each object in the order of mention prior to speech onset. Furthermore, greater processing difficulty increased the time speakers spent attending to each object. This demonstrates that the N2pc can track covert attention allocation in a naming task. In addition, an effect of processing difficulty at around 200-350 ms after stimulus onset revealed early attention allocation to the second to-be-named object. The flickering backgrounds elicited SSVEPs, but SSVEP amplitude was not influenced by processing difficulty. These results help complete the picture of the coordination of visual information uptake and motor output during speaking.
\end{abstract}

\section{Introduction}

Speaking seems easy, but successfully transforming a thought into speech, and saying the right words in the right order, requires the coordination of several complex processes (e.g., Bock and Levelt, 1994; Dell, 1986; Garrett, 1980; Fromkin, 1971). Speakers usually plan the first few words of an utterance and then begin to speak while planning the rest of the utterance, although the scope of advance planning depends on many factors (e.g., Costa and Caramazza, 2002; Konopka, 2012; Lee et al., 2013; Martin et al., 2010; Oppermann et al., 2010; Smith and Wheeldon, 1999; Wagner et al., 2010).

A particularly useful technique for addressing questions about how speakers coordinate processes involved in speaking is eye-tracking. In eye-tracking studies of language production, speakers typically describe displays featuring visually presented objects while their eye movements are recorded. Eye movements generally are a reliable reflection of the allocation of visual attention: they are directly preceded by attention shifts, and it is difficult, if not impossible, to move the eyes to one location and attend to a different location (Deubel and Schneider, 1996; Hoffman and Subramaniam, 1995). Focusing attention on an object likely facilitates the retrieval of associated information, including the object's name, suggesting that visual attention plays an important role in speaking (Griffin, 2004; Meyer and Lethaus, 2004). Indeed, eye movements have been found to be closely linked to speech planning processes (e.g., Griffin and Bock, 2000; Meyer et al., 1998). For instance, the speakers gaze at each object in the order of mention, and keep their gaze longer at objects associated with low frequency names than with high frequency names (Griffin, 2001; Meyer et al., 1998). Effects of word frequency and of phonological priming suggest that speakers shift gaze to the next object after having encoded the name of the previous object at the level of the phonological form (e.g., Meyer and van der Meulen, 2000). Thus, the eye movement record is thought to provide a window into the coordination of visual information uptake and motor output.

Of particular interest to the present study is evidence that, as speakers name each object in succession, to-be-named objects can be

\footnotetext{
* Correspondence to: Beckman Institute for Advanced Science and Technology, University of Illinois, 405 North Mathews Ave, Urbana, IL 61801, United States.

E-mail address: jrommers@illinois.edu (J. Rommers).
} 
processed to some extent before they have been fixated upon. Some of the evidence for this comes from gaze-contingent display changes (Pollatsek et al., 1984; Rayner, 1975). This is a technique in which, during the saccade from one object to the next, the object on which the saccade would have landed (the interloper) is replaced by a different object (the target). It has been observed that gaze durations on the target were shorter when the target and the interloper were identical, or each other's mirror image, or associated with the same name than when target and interloper were unrelated (Meyer et al., 2008; Morgan and Meyer, 2005; Schotter et al., 2013). This suggests that speakers processed the interloper prior to fixating on its location. Further evidence comes from studies where participants named pairs of objects that varied in processing difficulty, while the display remained constant. Gaze durations to the first object were shorter when the name of the second, not yet fixated, object was difficult to retrieve (and presumably interfered little with the retrieval of the name of the first object) than when the name of the second object was relatively easy to retrieve (Malpass and Meyer, 2010; see also Morgan et al., 2008).

Effects on processing of objects that have not been fixated upon likely arise because attention can be allocated to objects covertly, without moving the eyes. However, the nature and time course of covert attention shifts during speaking is unknown. This study examined whether additional information can be gleaned from direct measures of speakers' covert attention. These measures were derived from lateralized EEG activity, which has been well characterized in the attention literature.

\subsection{Electrophysiological signatures of covert attention allocation}

The EEG record is usually segmented into epochs, which are aligned in time and averaged point-by-point to create event-related potentials (ERPs), which are waveforms containing multiple components associated with various cognitive processes (for review, see Luck and Kappenman, 2011). In visual search tasks, shifting attention to a part of space in a display with multiple objects elicits an N2pc (N2 posterior-contralateral), a negativity that is larger over the hemisphere contralateral to an attended item than over the hemisphere ipsilateral to the attended item (Luck and Hillyard, 1990, 1994). The component occurs over lateral occipital electrode sites (dissociating it from other related components; Praamstra and Kourtis, 2010), usually begins around the time of the $\mathrm{N} 2$ wave, and has been associated with the allocation of spatial attention. In particular, because the N2pc is elicited when other distractor objects are present in the display, but not when distractors are absent, it has been associated with attentional filtering processes that suppress competing information in the environment (Luck and Hillyard, 1994). Although there is some controversy regarding the exact functional interpretation of the N2pc in terms of distractor suppression versus target processing (e.g., Eimer, 1996; Hickey et al., 2009; Mazza et al., 2009), or more generally competition resolution (Luck, 2012), there is broad consensus that the N2pc indexes covert attention allocation. Because the EEG is a direct reflection of neural activity, the onset latency of the N2pc can be interpreted as the latest moment at which attention was focused on an item.

Another electrophysiological signature of covert attention allocation is the phenomenon of steady-state visual evoked potentials (SSVEPs), which are continuous oscillatory responses at the frequency of regularly repeating visual stimuli (Regan, 1989; Wilson and O'Donnell, 1986; for review, see Vialatte et al., 2010). SSVEPs have been used to track attention allocation in sustained attention paradigms, in which a different visual stimulus is shown in each visual field, and participants attend to the stimuli in one hemifield for the duration of a block of trials. The stimuli, or small background squares on which the stimuli are superimposed, flicker at different frequencies, eliciting SSVEPs at each frequency. Importantly, it has been observed that the SSVEP amplitude is greater for attended than unattended locations (Morgan et al., 1996; Müller et al., 1998a). In addition, when a central cue directs attention to a particular stimulus on a trial-by-trial basis, SSVEPs evoked by the attended stimulus also increase in amplitude (Müller et al., 1998b). SSVEPs have been interpreted as reflecting a gain-control mechanism that enhances discriminability by increasing the signal-to-noise ratio of attended stimuli (Müller et al., 1998b). An advantage of SSVEPs is that, whereas the N2pc is a contralateral minus ipsilateral difference waveform and therefore a relative measure of attention across both hemifields, SSVEP "frequency tagging" allows for tracking the allocation of attention to individual objects separately. In sum, at least two measures of covert attention allocation have been documented that could help elucidate the nature and time course of attention shifts during speaking.

\subsection{The present study}

This study recorded EEG while speakers described pairs of objects using short utterances, such as "dog and chair". Speakers named the objects from left to right or from right to left depending on the objects' colors, while fixating on a central fixation cross. The objects were superimposed onto background squares which flickered at different frequencies. This allowed for addressing several related questions.

First, we examined whether the N2pc and SSVEPs are detectable when speakers name pairs of objects. One hurdle in the electrophysiology of language production is the contamination of the signal by muscle activity, which can affect almost all electrodes on the scalp (Goncharova et al., 2003; for experimental paradigms that avoid speech muscle activity, see Habets et al., 2008; Jescheniak et al., 2002; van Turennout et al., 1998). Such muscle activity could reduce the signal-to-noise ratio, making it more difficult to observe the effects of interest.

However, there are several reasons to assume that the signals of interest will be detectable. By now, a number of studies has recorded EEG in overt speech production tasks (for examples and review, see Eulitz et al., 2000; Ganushchak et al., 2011; Piai et al., 2012; Strijkers et al., 2011). The N2pc and SSVEPs have the advantage of being distributed over electrode sites contralateral to the direction of attention, such that motor activity should cancel out after collapsing across left-to-right and right-to-left naming directions. While the N2pc has to our knowledge not previously been applied to language production research, it has reliably been observed both in typical visual search studies, in which a target is displayed along with many distractor objects, and in studies with displays more similar to ours, in which a target is presented along with a single distractor (Eimer, 1996). Furthermore, SSVEPs have been recorded in a great variety of situations (Vialatte et al., 2010) and also concurrently with the N2pc (Müller and Hillyard, 2000). Thus, we hypothesized that our paradigm would elicit an N2pc and SSVEPs.

We further investigated whether, like overt attention in the form of eye movements, covert attention can also provide a window into the coordination of visual information uptake and motor output. If so, covert attention as reflected by the N2pc and SSVEPs would be expected to shift to the first to-be-named object after the onset of the stimuli, followed by a shift to the second to-be-named object.

The evidence discussed above, that the second to-be-named object can be processed before it has been fixated upon, raises certain hypotheses concerning covert attention, but these hypotheses are difficult to evaluate using eye movements alone. Direct and continuous measures of covert attention allocation could significantly improve our understanding of the time course of processing objects in a naming task. Thus, in addition, we orthogonally varied the processing difficulty of each object by presenting it in upright orientation (easy) or in upside-down orientation (difficult). This manipulation has clear effects on naming latencies (Malpass and Meyer, 2010) without affecting the visual complexity of the pictures. This enabled us to examine at what points in time properties of each object influenced the allocation of attention, in particular, whether the effects of first object difficulty and 
second object difficulty would occur at an early or a late point in time.

\section{Methods}

\subsection{Participants}

Twenty-four native speakers of Dutch (19 female, 5 male; mean age 21 years, range 18-24 years) gave informed consent and were paid to take part in the study. All were right-handed and had normal or corrected-to-normal vision and no history of language-related or neurological disorders. Data from five additional participants were excluded because less than $60 \%$ of the trials was left after removing trials with naming errors and applying our relatively stringent artifact rejection thresholds (see EEG analysis). One additional participant was excluded because they did not complete the experiment. The study had been approved by the regional ethics review board.

\subsection{Materials and design}

The stimuli consisted of 112 line drawings of common objects (see Appendix). A pilot study had established that they were easy to recognize and name. Each line drawing was cropped such that the various objects occupied a similar amount of space within the square boundaries and then scaled to 200 by 200 pixels.

The processing difficulty of the two objects was orthogonally varied by presenting each object in upright orientation or upside down, yielding four conditions: EasyEasy (both objects in upright orientation), EasyDifficult (with the second object upside down), DifficultEasy (with the first object upside down), and DifficultDifficult (both objects upside down). An example is shown in Fig. 1. Only objects for which a canonical orientation existed were used, such that viewing them in upright orientation versus upside down made a difference (for example, a giraffe was included, whereas a ball was not). The objects' line colors were edited to indicate which object to name first (green) and which object to name second (red). To discourage participants from attending to one side of the screen prior to the presentation of the objects, the red and green objects' positions were varied such that on half of the trials the naming order was left-to-right and on the other half of the trials the naming order was right-to-left.

The objects were combined into a set of 448 pairs, avoiding semantic and phonological relationships between their names. Across all object pairs, each individual object was used eight times, once in each of the eight possible combinations of the two colors and the four object difficulty conditions. Object combinations were not repeated so that on a given trial the identity of one object was not predictive of the identity of the other object. Object pairs were rotated across difficulty conditions such that, across four lists, each unique pair of objects occurred in every condition. Lists were pseudorandomized individually for each participant, under the constraints that trials featuring objects of the same semantic category did not occur in immediate succession, and that the same object difficulty condition did not occur on more than four trials in a row.

\subsection{Procedure}

First, participants were familiarized with the object names by naming all 112 experimental objects and eight additional objects that would appear in the practice session in random order. Each object was presented for three seconds, in black line color on a light grey background. After the object had been on the screen for $600 \mathrm{~ms}$, a written word corresponding to the object's name was overlaid onto the object. Participants named each object using the written word. When all objects had been named once, they appeared again in the same order but without the written words, and participants named the objects again. They were notified of any mistakes. Participants then practiced naming pairs of objects for a block of sixteen trials featuring only the eight non-experimental objects that they had been familiarized with. The practice block was repeated up to three times, until the EOG signal suggested successful maintenance of fixation on the center of the screen and naming accuracy was acceptable.

The 448 experimental trials were presented in 28 blocks of 16 trials. On each trial, a white central fixation cross was presented on a black background and remained on the screen throughout the trial. After $800 \mathrm{~ms}$, two squares appeared that subtended 5 degrees of visual angle each, placed at 3.1 degrees eccentricity (following recommended settings for SSVEPs; Ng et al., 2012). The squares began flickering by flashing white for the duration of one frame $(\sim 13 \mathrm{~ms}$, given a $75 \mathrm{~Hz}$ screen refresh rate). Flashes occurred once every third frame on one side $(25 \mathrm{~Hz})$ and once every fourth frame on the other side $(18.75 \mathrm{~Hz}$; counterbalanced between participants), forming a 12-frame sequence which kept repeating throughout the trial. After $1600 \mathrm{~ms}$, a red and a green object appeared and remained on the screen for $3200 \mathrm{~ms}$,
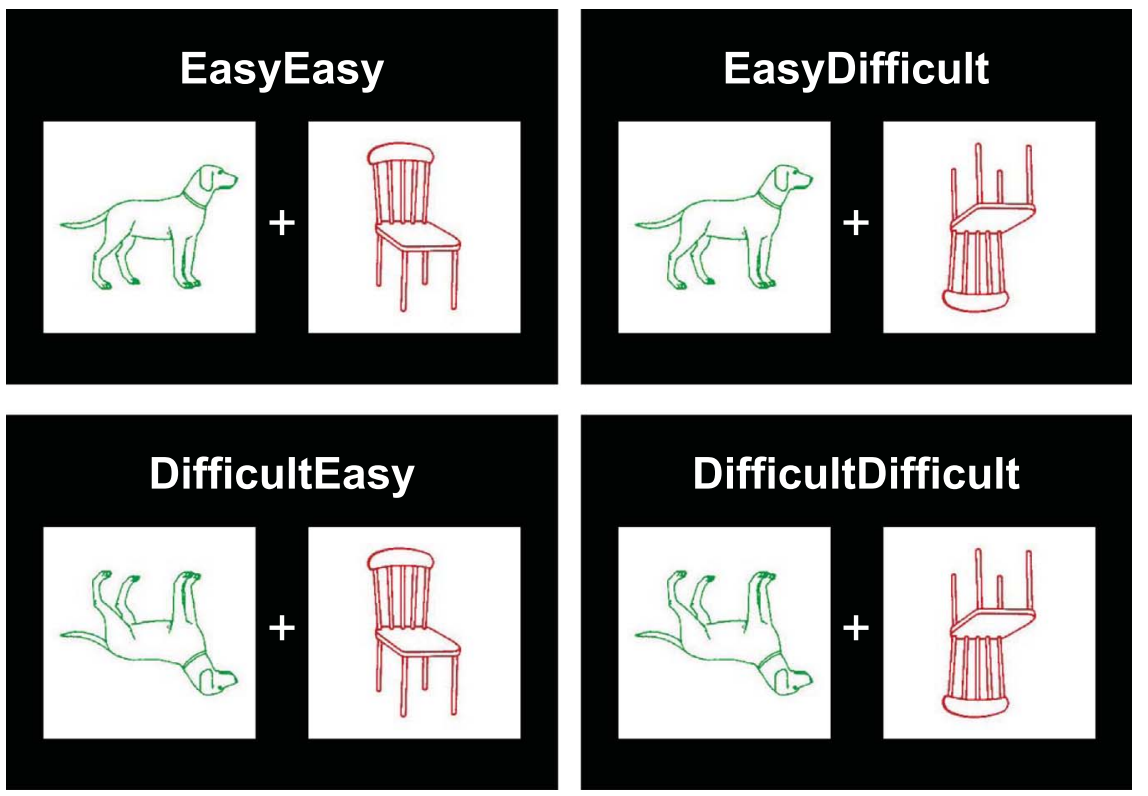

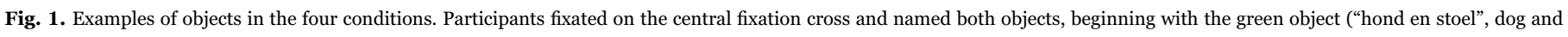

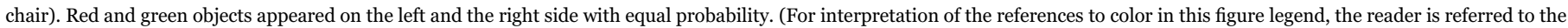
web version of this article.) 
superimposed onto the flickering squares. Participants named the objects in the structure "[green object] and [red object]" (e.g. "dog and chair") and their naming latencies were recorded using a softwarebased voice key. Finally, three asterisks $(* * *)$ appeared in the center of the screen for $1 \mathrm{~s}$, indicating that participants were free to blink. After every block, participants had the opportunity to take a break.

\subsection{EEG recording and preprocessing}

The electroencephalogram was recorded from 61 active $\mathrm{Ag} / \mathrm{AgCl}$ electrodes mounted in a carefully positioned cap (actiCAP) according to an equidistant montage, referenced to the left mastoid. Two additional electrodes were placed on the orbicularis oris muscle above and below the left corner of the mouth. The ground electrode was placed on the forehead. Electrode impedance was kept below $20 \mathrm{k} \Omega$. The recordings were amplified through BrainAmp DC amplifiers with a bandpass filter of $0.016-100 \mathrm{~Hz}$ and digitized on-line with a sampling frequency of $500 \mathrm{~Hz}$.

The following preprocessing steps were common to all EEG analyses. The data were re-referenced off-line to the average of the left and right mastoids. Bipolar horizontal EOG was computed as the difference between the signals from two electrodes placed at each outer canthus. Taking into account that the longest recorded reaction time was $2500 \mathrm{~ms}$, and adding padding for time-frequency analyses, the continuous EEG was segmented into relatively long epochs spanning from -1320 to $3500 \mathrm{~ms}$ around the onset of the visually presented objects. A pre-stimulus baseline of $320 \mathrm{~ms}$ (two 12-frame flickering cycles) was subtracted.

Artifacts were rejected using participant-specific thresholds which considered the combination of speaking-related muscle activity and potential eye movements present in the data set. In data that have been averaged across trials, speaking-related muscle activity should largely cancel out when comparing contralateral and ipsilateral activity taken from the same electrodes, under the reasonable assumption that the topographical distribution of speaking-related muscle activity will not co-vary with the direction of attention (see also Results Fig. 4, bottom panels). However, on individual trials, speaking-related motor activity may be asymmetrically distributed and cause deflections in the bipolar horizontal EOG. To reduce the influence of such speaking-related muscle activity on single-trial eye movement estimates, a two-pass 4th order $20 \mathrm{~Hz}$ low-pass Butterworth filter was applied to the horizontal EOG channel. Careful inspection confirmed that eye movements remained clearly visible after filtering. Artifact rejection then proceeded in two time windows. In a window from -320 to $600 \mathrm{~ms}$ relative to stimulus onset, where relatively little speakingrelated motor activity was expected, trials were rejected if the horizontal EOG exceeded $30 \mu \mathrm{V}$ (or if the scalp channels showed blinks, drifts, or other artifacts). A participant's data were kept if after removal of these epochs at least two thirds of the trials remained and the HEOG did not exceed $4 \mu \mathrm{V}$ at any time sample after averaging across trials (similar to Woodman and Luck, 2003b). This corresponds to $\sim 0.25^{\circ}$ and a propagated voltage of $\sim 0.1 \mu \mathrm{V}$ at posterior channels (Lins et al., 1993). For the remainder of the epoch (600-2500 ms), participant-specific thresholds removed drifts and eye blinks observed on scalp channels, while retaining the speaking-related motor activity that was part of the task. Trials with eye movements were removed by applying moving window peak-to-peak thresholds on the horizontal EOG channel. In all, this relatively strict procedure removed $31 \%$ of the trials, with similar trial numbers remaining across conditions (Mean \pm SD: EasyEasy $78 \pm 14$; EasyDifficult $77 \pm 11$; DifficultEasy $78 \pm 17$; DifficultDifficult $75 \pm 13$ ).

\subsection{Analysis}

\subsubsection{Behavioral data}

The behavioral data were analyzed using mixed-effects regression models which simultaneously take into account items and participants as random effects (Baayen et al., 2008). Items were defined as object pairs. Naming accuracy (correct, incorrect) was analyzed using a logistic linking function, whereas the continuous naming latencies for correctly named trials were analyzed using a linear model. Naming latencies were log-transformed to reduce skewness. ANOVA-style deviation coding was used (Easy: -0.5 ; Difficult: 0.5), making the intercept interpretable as the grand mean and the other terms interpretable as main effects and an interaction. We initially attempted to fit by-participant and by-item random intercepts and random slopes for the main effect of Object 1 Difficulty, the main effect of Object 2 Difficulty, and the interaction of Object 1 and Object 2 Difficulty (the maximal random effects structure warranted by the design; Barr et al., 2013), but simplified the models if they failed to converge by iteratively removing the term that explained the least variance. $P$ values for a given fixed effect were derived from likelihood ratio tests which compared the model to an otherwise identical model without the fixed effect of interest.

\subsubsection{Event-related potentials}

Event-related potentials were obtained by averaging trials in the time domain within each condition. A two-pass 4th order Butterworth $15 \mathrm{~Hz}$ low pass filter was applied to the ERPs to reduce the amplitude of the steady-state visual evoked potentials. The N2pc was calculated as the difference between occipital electrode sites contralateral and ipsilateral to Object 1 (highlighted in Results Fig. 4; relative to the $10 \%$ system, the left channel is positioned between the sites P7 and PO7, and the right channel between the sites P8 and PO8). In the absence of prior knowledge about the time course of the effects of interest, statistically significant differences between conditions were identified using nonparametric cluster-based permutation tests (Maris and Oostenveld, 2007) implemented in the Matlab toolbox Fieldtrip (Oostenveld et al., 2011). Briefly, the procedure is as follows. First, a dependent-samples $t$-test compares the conditions at every data point (in this case, time sample), and data points that do not meet a significance level of 0.05 are zeroed. Adjacent non-zero data points are combined into clusters for each of which the cluster-level $t$-value is the sum of all $t$-values within the cluster, and the cluster with the greatest sum is selected. Then a null-distribution is created by randomly assigning subject averages to one of the two conditions 1000 times and computing the cluster-level statistics for each randomization. Finally, the observed cluster-level test statistics are compared against the null-distribution. When the observed statistic falls in one of the 2.5th percentiles of the null-distribution, the effect is considered significant. Note that this test can only compare two conditions at a time. In order to base our inferences about effects of object difficulty on as large a number of trials as possible, we averaged across pairs of conditions. Thus, the effect of Object 1 Difficulty was quantified, like a main effect, as the average across the two conditions with a difficult first object (DifficultDifficult and DifficultEasy) versus the average of the two conditions with an easy first object (EasyEasy and EasyDifficult). Similarly, the effect of Object 2 Difficulty was quantified as the average across the two conditions with a difficult second object (EasyDifficult and DifficultDifficult) versus the average across the two conditions with an easy second object (EasyEasy and DifficultEasy).

Because the timing inferences one can draw from cluster-based permutation tests are limited (Maris, 2012), a jackknifed fractional peak latency analysis quantified N2pc timing differences between conditions. The fractional peak latency is computed from a peak in the signal, back in time, as the point at which the voltage reached a certain fraction of the peak, such as $50 \%$. The jackknife technique, introduced to ERP research by Miller et al. (1998), involves creating a leave-one-out grand average for each participant, to which all participants except the participant in question contribute. Leave-one-out averages are less noisy than individual participant ERP waveforms, making it easier to measure the fractional peak latency. The jackknifed leave-one-out averages can be subjected to analyses of variance (ANOVAs) with an adjustment to the degrees of freedom (Ulrich and Miller, 2001). Alternatively, as done in this study, an unadjusted ANOVA can be applied to the individual participant latencies, which 
can be retrieved from the jackknifed subaverages via a simple formula (Smulders, 2010).

For the jackknifed fractional peak latency analysis, the N2pc waveforms were low pass filtered with a half-amplitude cutoff at $10 \mathrm{~Hz}$ (Luck, 2005, p. 246), using a 100th order finite impulse response filter. Because the N2pc was expected to show both a shift of attention to Object 1 and a shift to Object 2, the jackknifed fractional peak latency was measured for the most negative peak and the most positive peak in the epoch. The onset of the negativity was defined as the last sample before the negative peak that was equal to or less negative than $50 \%$ of the peak amplitude (a setting known to yield high power; Kiesel et al., 2008). The onset of the positivity was defined as the last sample before the positive peak that was equal to or less positive than $50 \%$ of the peak amplitude.

\subsubsection{Steady-state visual evoked potentials}

Trials from each condition were averaged in the time domain. SSVEP power over time was then computed using a sliding time window Fast Fourier Transform approach at the two flickering frequencies. The window was $480 \mathrm{~ms}$ long (fitting 12 cycles of a $25 \mathrm{~Hz}$ oscillation, or 9 cycles of an $18.75 \mathrm{~Hz}$ oscillation) and moved along the time axis in steps of $10 \mathrm{~ms}$. Each instance of a time window was multiplied with a Hanning taper and Fourier-transformed. For each subject and each flickering frequency, the maximal SSVEP channel was selected based on power averaged across all conditions in a pre-stimulus time window from -1000 to $-320 \mathrm{~ms}$ relative to the onset of the two objects (when the backgrounds were already flickering). The SSVEP channels were selected from a set of eight left and eight right posterior channels contralateral to the flickering stimulus (including two midline channels; see results in Fig. 5). SSVEP power over time at these channels was subjected to cluster-based permutation tests in the same way as the N2pc was. Effects of Object 1 Difficulty and Object 2 Difficulty were examined separately for SSVEP power at the flickering frequency of Object 1 and at the flickering frequency of Object 2.

\section{Results}

\subsection{Behavioral results}

Average naming accuracy is shown in the left panel of Fig. 2. Objects were named more accurately by an average of $\sim 3 \%$ when presented in upright orientation than when presented upside-down. The regression models with a maximal random effects structure did not converge, even when omitting random correlations and random intercepts from all random effects terms. Models did converge after omitting the random slope for the interaction; estimates of the interactions may therefore be considered anti-conservative (Barr et al., 2013). Accuracy decreased both when Object 1 was difficult, $\beta=0.27468, S E=0.08915, z=3.081, \chi^{2}=7.0083, p=0.008$, and when Object 2 was difficult, $\beta=0.43546, S E=0.08337, z=5.223, \chi^{2}=18.388$, $p<0.001$. Evidence for an interaction between Object 1 Difficulty and Object 2 Difficulty was not strong, $\beta=-0.22022, S E=0.13946$, $Z=-1.579, \chi^{2}=2.2568, p=0.113$.

As shown in the right panel of Fig. 2, speakers began their utterances earlier when Object 1 was easy than when it was difficult, $\beta=0.055799$ (64 ms), $S E=0.005433, t=10.27, \chi^{2}=47.868, p<0.001$. The difficulty of Object 2 did not affect naming latencies, $\beta=0.002052$ ( $2 \mathrm{~ms}$ ), $S E=0.023329, t=0.09, \chi^{2}=0.1883, p=0.664$, and neither was there an interaction between Object 1 Difficulty and Object 2 Difficulty, $\beta=-0.008585$ ( $-10 \mathrm{~ms}$ ), $S E=0.008210, t=-1.05, \chi^{2}=1.1026, p=0.294$.

One may wonder whether the left-to-right versus right-to-left naming order affected the naming latencies. An exploratory analysis added Naming Order to the model, which converged after simplification, retaining the fixed effects of Object 1 Difficulty, Object 2 Difficulty, Naming Order, and only the Object 2 Difficulty $\times$ Naming Order

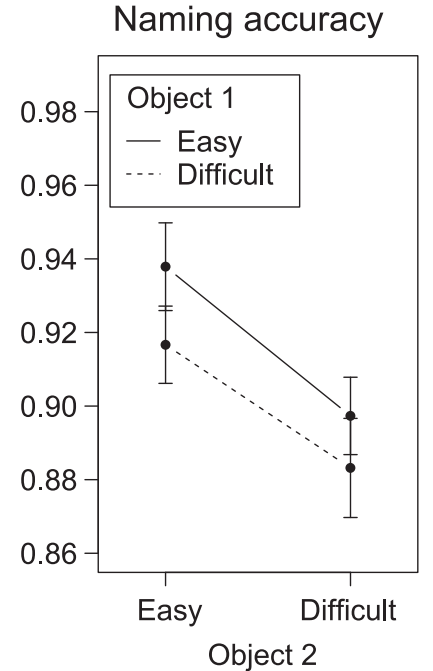

Naming latencies (ms)

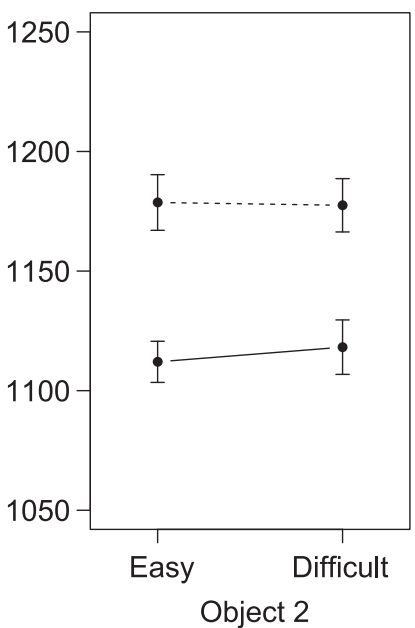

Fig. 2. Behavioral results. The left panel shows the naming accuracy proportions; the right panel shows the naming latencies for correctly named objects. Error bars indicate within-participants corrected 95\% confidence intervals of the participant means (Morey, 2008).

interaction, by-participant random intercepts and slopes for Object 2 Difficulty and Naming Order, and by-item random slopes for Object 1 Difficulty, Naming Order, and the Object 2 Difficulty $\times$ Naming Order interaction. The model confirmed the effect of Object 1 Difficulty, $t=12.11$, and the absence of an effect of Object 2 Difficulty, $t=0.53$, as reported above. There was no main effect of Naming Order, $\beta=-0.004884 \quad(-5 \mathrm{~ms}), \quad S E=0.010870, \quad t=-0.45, \quad \chi^{2}=0.2045$, $p=0.6511$, but there was an Object 2 Difficulty $\times$ Naming Order interaction, $\beta=-0.018256, S E=0.008314, t=-2.20, \chi^{2}(1)=4.8169$, $p=0.02818$. Follow-up analyses revealed that the interaction arose from a marginal effect of Object 2 Difficulty (longer latencies when Object 2 was difficult than when it was easy) when naming left-to-right, $\beta=0.010786$ (12 ms), $S E=0.006114, t=1.76, \chi^{2}=3.1039, p=0.0781$, combined with a numerical difference in the opposite direction when naming right-to-left, $\beta=-0.007689(-8 \mathrm{~ms}), S E=0.006260, t=-1.23$, $\chi^{2}=1.5092, p=0.2193$.

Because naming order is confounded with the visual hemifield in which each object was presented, this subtle effect is difficult to interpret. One possibility is that the second object affected naming latencies more when naming left-to-right than when naming right-toleft; another possibility is that the second object had a greater impact on naming latencies when it was presented in the right visual field (projecting onto the left hemisphere) than when it was presented in the left visual field. Note that for the EEG analyses data were collapsed across left-to-right and right-to-left naming orders to eliminate any such differences.

\subsection{Event-related potentials}

The grand average ERP waveforms time-locked to the onset of the two objects are shown in Fig. 3. Note that, in this two-object paradigm, contralateral to Object 2 is equivalent to ipsilateral to Object 1 , and vice versa. After the P1 and N1, a slow negative-going wave was initially more negative contralateral to Object 1 than contralateral to Object 2 (ipsilateral to Object 1), indicating a shift of attention to Object 1 . This amplitude relationship reversed after about $1 \mathrm{~s}$, indicating a shift of attention to Object 2. The underlying ERP component causing the changes in amplitude relationship is the N2pc.

The statistical analyses of the waveforms contralateral to Object 1 versus the waveforms contralateral to Object 2 (or ipsilateral to Object 1) confirmed the presence of effects of positive and negative polarity in each condition; EasyEasy, negative cluster from 192 to $734 \mathrm{~ms}$, 

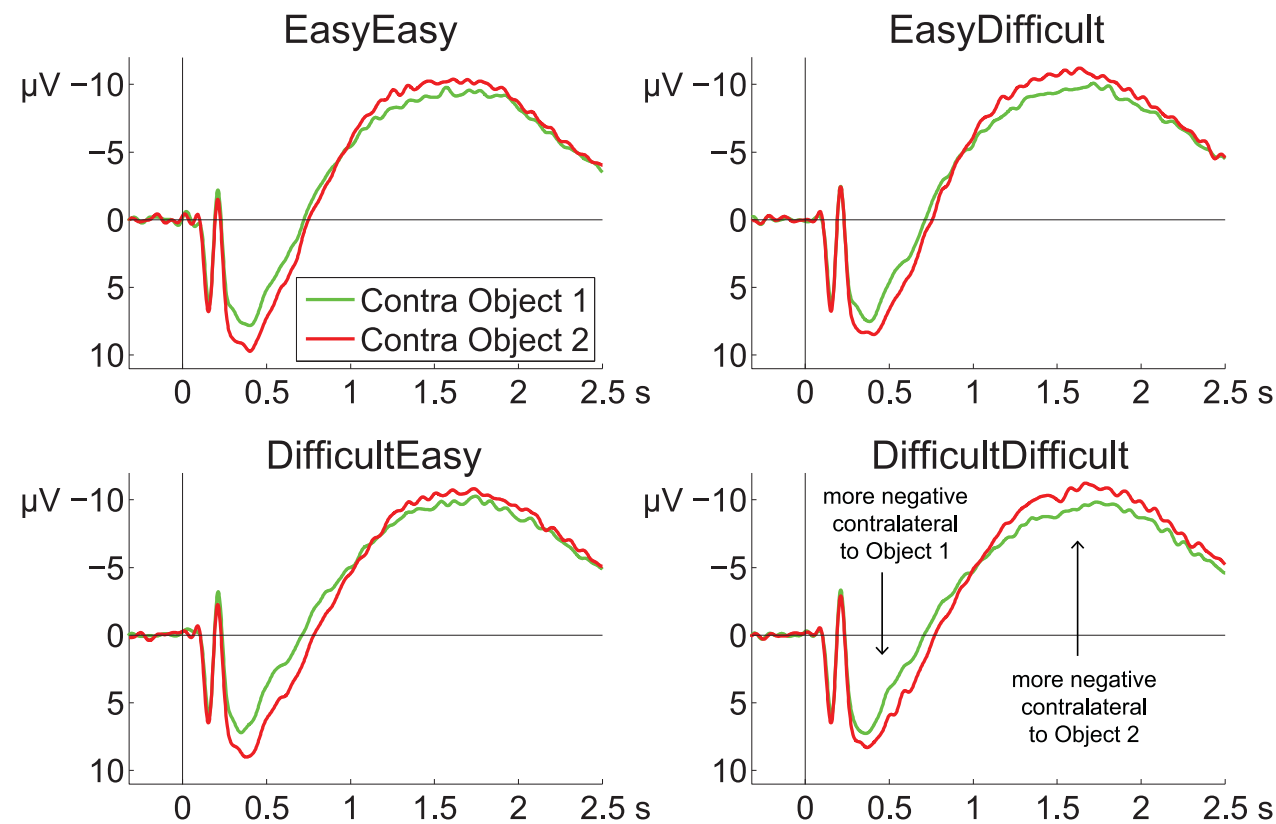

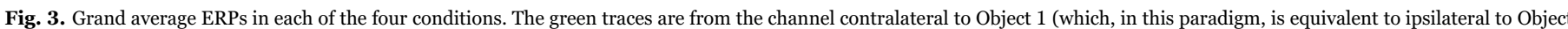

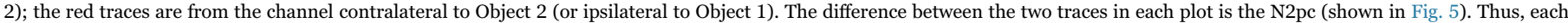

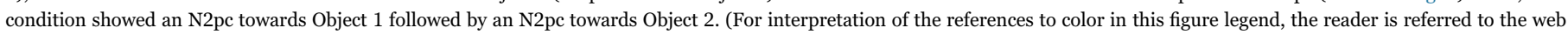
version of this article.)

$p=0.002$, positive cluster from 1150 to $1556 \mathrm{~ms}, \quad p=0.002$; EasyDifficult, negative cluster from 260 to $802 \mathrm{~ms}, p=0.002$, positive cluster from 1034 to $1720 \mathrm{~ms}, p=0.004$; DifficultEasy, negative cluster from 200 to $944 \mathrm{~ms}, p=0.002$, positive cluster from 1406 to $1536 \mathrm{~ms}$, $p=0.074$; DifficultDifficult, negative cluster from 246 to $918 \mathrm{~ms}$, $p=0.002$, positive cluster from 1146 to $2168 \mathrm{~ms}, p=0.002$.

As shown in Fig. 4, the N2pc had a typical lateral occipital distribution, which was visible after subtracting ipsilateral from contralateral activity, to isolate the $\mathrm{N} 2 \mathrm{pc}$ from the slow negative-going wave. The distribution of the N2pc was essentially identical prior to speech onset and during speaking.

The reversal of the amplitude relationship can also be seen in difference waveforms of activity contralateral minus ipsilateral to Object 1, shown in Fig. 5, where a negativity indicates the shift to Object 1 , followed by a positivity indicating the shift to Object 2 . Waveforms were averaged across conditions representing the Difficult and Easy levels of the factors Object 1 Difficulty and Object 2 Difficulty.

As shown in the upper panel of Fig. 5, compared with when Object 1 was easy to process, when Object 1 was difficult, the positive-going difference wave (or attention shift to Object 2) was delayed. Statistically, this was reflected in a cluster from 666 to $1110 \mathrm{~ms}$, $p=0.004$. As shown in the lower panel of Fig. 5, increased Object 2 Difficulty attenuated the amplitude of the initial negativity (or attention shift to Object 1 ). This was reflected in a briefly interrupted cluster from 206 to $326 \mathrm{~ms}, p=0.016$, and 352 to $440 \mathrm{~ms}, p=0.020$. Object 2 Difficulty also increased the amplitude of the later positivity, as confirmed statistically by a cluster from 1530 to $1676 \mathrm{~ms}, p=0.016$.

The jackknifed $50 \%$ fractional peak latency analyses confirmed the timing differences. The onset of the attention shift to Object 1 (EasyEasy: $235 \mathrm{~ms}$, EasyDifficult: $391 \mathrm{ms,} \mathrm{DifficultEasy:} 230 \mathrm{~ms}$, DifficultDifficult: $381 \mathrm{~ms}$ ) occurred earlier by about $150 \mathrm{~ms}$ when Object 2 was easy than when Object 2 was difficult, $F(1,23)=27.912$, $p<0.0001$. There was no effect of Object 1 difficulty on the shift to Object $1, F(1,23)=0.124, p=0.728$ ( $-7 \mathrm{~ms}$ difference), and no interaction, $F(1,23)=0.013, p=0.909$. Conversely, the shift to Object 2 (EasyEasy: $1024 \mathrm{~ms}$, EasyDifficult: $1024 \mathrm{~ms}$, DifficultEasy: $1219 \mathrm{~ms}$, DifficultDifficult: $1139 \mathrm{~ms}$ ) occurred earlier by $155 \mathrm{~ms}$ when Object 1 was easy than when Object 1 was difficult, $F(1,23)=20.844, p<0.0002$.

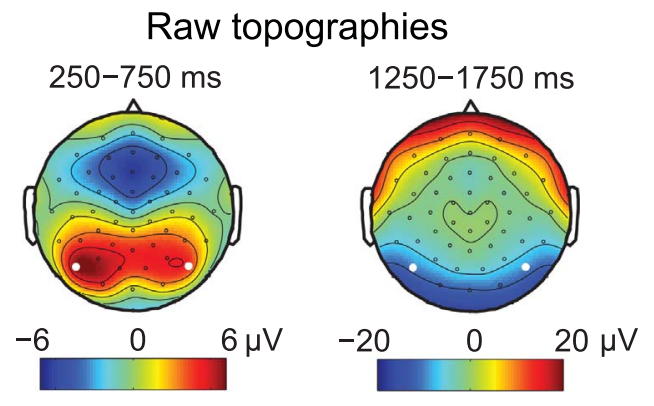

N2pc topographies

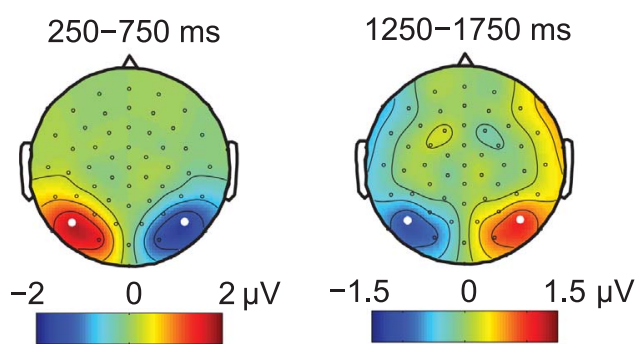

Fig. 4. Grand average topographies averaged across all conditions, for the time windows during which the shift to Object 1 and to Object 2 occurred. Top: the data were arranged such that channels ipsilateral to Object 1 appear on the left and the channels contralateral to Object 1 appear on the right, thus normalizing to a left-to-right naming direction. Bottom: the N2pc topographies, showing the contralateral minus ipsilateral difference for each "homologous" electrode pair, projected onto both hemispheres with opposite polarity. White dots indicate the N2pc channels that were used for analysis.

There was no clear evidence for an effect of Object 2 difficulty on the timing of the shift to Object $2, F(1,23)=2.270, p=0.146(40 \mathrm{~ms}$ difference), although an amplitude difference had been detected by the cluster-based permutation test later in the epoch. There was no interaction, $F(1,23)=1.608, p=0.217$. In sum, in contrast to the naming latencies, N2pc amplitude and latency were affected not only by Object 1 difficulty but also by Object 2 difficulty, and these effects occurred at different points in time. 

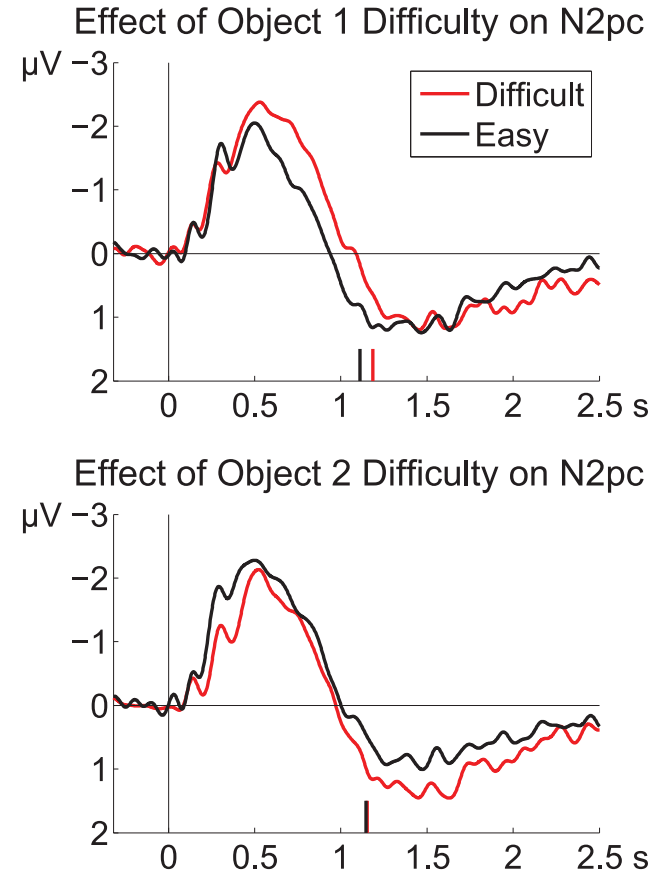

Fig. 5. Grand average N2pc waveforms of activity contralateral to Object 1 minus contralateral to Object 2, averaged across conditions representing the difficult (red lines) and easy (black lines) levels of the factors Object 1 Difficulty (upper panel) and Object 2 Difficulty (lower panel). Negative values indicate greater negativity contralateral to Object 1, whereas positive values indicate greater negativity contralateral to Object 2 (or ipsilateral to Object 1). The vertical lines on the time axis indicate average speech onsets. (For interpretation of the references to color in this figure legend, the reader is referred to the web version of this article.)

\subsection{Steady-state visual evoked potentials}

The flickering backgrounds elicited SSVEPs, of which the topographical distribution prior to the onset of the objects is shown in Fig. 6. SSVEPs had an occipital distribution, right-lateralized at the left flickering frequency, and left-lateralized at the right flickering frequency (though relatively less strongly, possibly due to a left-to-right naming preference). Individual participant topographies (not shown) exhibited more focal, often nonoverlapping, maxima for the two flickering frequencies.

The time course of the steady-state visual evoked potentials is shown in Fig. 7. Overall, power dropped after onset of the two objects at time zero (possibly reflecting a reduction in flickering contrast because of the superimposed objects). SSVEP power then remained relatively stable. Analogously to the N2pc analysis, effects of object difficulty were evaluated, but now for the individual objects SSVEPs, that is, at the flickering frequency of Object 1 and at the flickering frequency of Object 2 separately.

For SSVEP amplitude at the frequency of Object 1, shown in the left
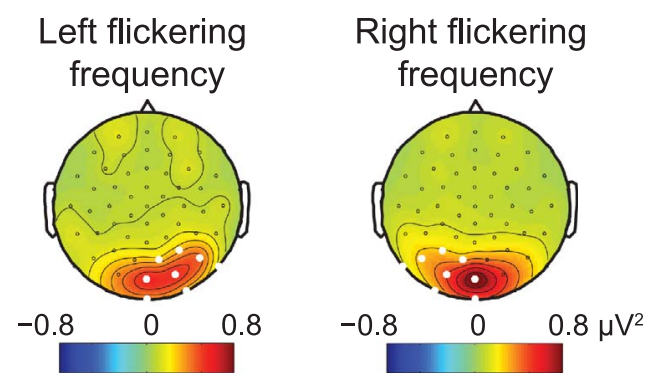

Fig. 6. Grand average SSVEP distribution from -1000 to $-320 \mathrm{~ms}$ relative to objects onset, for the frequency corresponding to the left flickering square and for the frequency corresponding to the right flickering square. White dots indicate channels that were considered when selecting SSVEP channels for individual participants. panels of Fig. 7, there was no effect of Object 1 Difficulty, $p=0.236$, or of Object 2 Difficulty, all $p>0.513$. For SSVEP amplitude at the frequency of Object 2, shown in the right panels of Fig. 7, there was also no effect of Object 1 Difficulty (no clusters), or of Object 2 Difficulty, $p=0.252$. In sum, while the flickering backgrounds successfully elicited SSVEPs, there was no evidence that SSVEP amplitude was modulated by the difficulty of processing the objects.

\section{Discussion}

This study measured electrophysiological signatures of covert attention allocation as speakers described pairs of objects. The main questions were whether an N2pc and SSVEPs would be observed, whether these signals would reflect the order of mention and the difficulty of processing the objects, and if so, what the time course of the allocation of covert attention would be.

An ERP analysis revealed that, despite distortion from muscle artifacts inherent to EEG signals recorded during speech production, the speakers' attention shifts were detectable in the form of a clear N2pc, not only prior to speech onset, but also during articulation. This demonstrates the viability of using EEG to address questions about covert attention allocation in language production, and attests to the robustness of this ERP component. The detection of covert attention shifts was likely afforded in part by the N2pc's occipital distribution and the contralateral nature of the component, which isolates it from muscle activity and other parts of the signal whose topography does not vary as a function of the direction of attention. Because direct comparisons between conditions would be influenced by differences in speech motor activity, we only compared contralateral and ipsilateral channels within conditions, or N2pc difference waves between conditions.

Across all conditions, the N2pc pattern reflected the objects' order of mention. In the easiest condition, the N2pc showed an initial attention shift to Object 1 around $230 \mathrm{~ms}$ after display onset, followed by a shift to Object 2 around $1000 \mathrm{~ms}$ (note that, because the data were filtered, these estimates are imprecise). Both shifts were observed prior to average speech onset, which occurred around $1100 \mathrm{~ms}$. Because the second N2pc occurred later than in visual search studies, one may wonder whether it is the same component, and whether it still indexes covert attention. However, previous work has also observed temporally sustained N2pcs, as well as two N2pcs of opposite directions occurring in succession (Woodman and Luck, 2003b). The N2pc is the only known component that can explain the observed difference between contralateral and ipsilateral channels, and the scalp distributions of the first and the second component were highly similar. It therefore seems likely that, analogously to overt attention as indexed by eye movements, the type of covert attention captured by the N2pc can provide a window into the coordination of visual information uptake and motor output during speaking.

By presenting each object in upright or upside down orientation, we could assess the influence of each object's processing difficulty on the allocation of attention over time. Compared with when Object 1 was easy, a difficult Object 1 delayed the N2pc shift to Object 2 by about $150 \mathrm{~ms}$, at a time around 700-1100 ms after the objects' onset. This suggests that processing difficulty extended the time speakers spent attending to Object 1 . Similarly, after the attention shift to Object 2 had occurred, the second N2pc was greater in amplitude for difficult second objects than for easy second objects. Thus, consistent with the overall N2pc effects across conditions, the processing difficulty of each object affected attention allocation when that object was being attended to.

However, the effects of processing difficulty did not fully follow the objects' order of mention. Strikingly, the difficulty of Object 2 affected the N2pc not only after the attention shift to Object 2, but also at a much earlier point in time, around 200-350 ms after the display onset. Overall, the N2pc at this point in time indicated a relative shift toward Object 1, but this shift occurred later, or was initially less pronounced, 
Object 1: Object 1 Difficulty

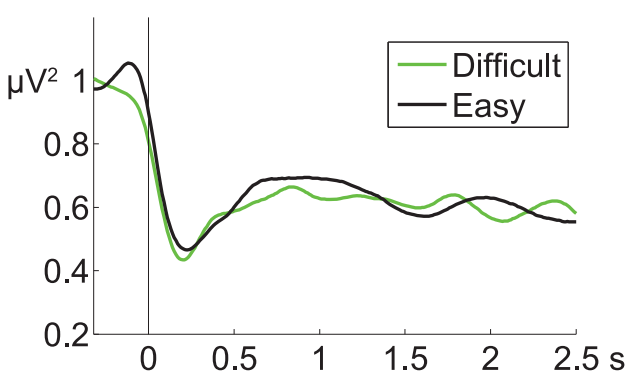

Object 2: Object 1 Difficulty

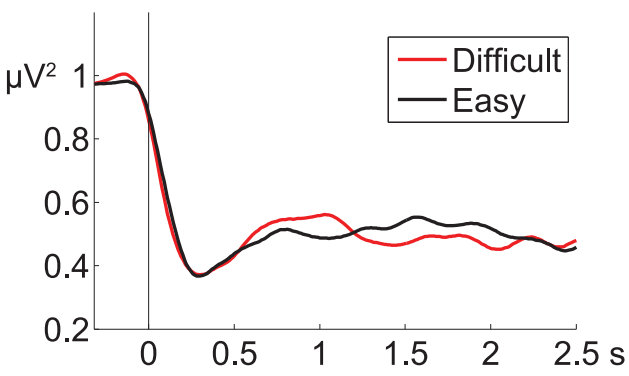

\section{Object 1: Object 2 Difficulty}
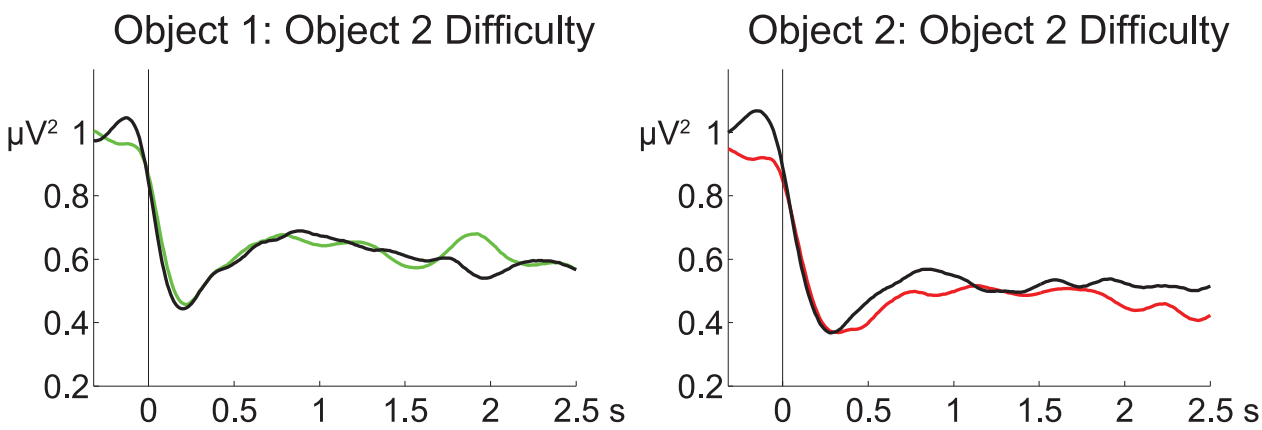

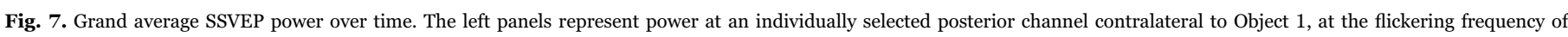

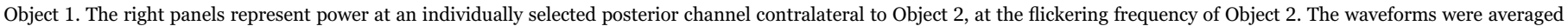
across conditions representing the difficult and easy levels of the factors Object 1 Difficulty (upper panels) and Object 2 Difficulty (lower panels).

when Object 2 was difficult than when it was easy. This suggests very early processing of Object 2 . Such an effect was not observed in the naming latencies in this study, which only revealed an effect of Object 1 difficulty, nor had it been observed in eye movements in previous studies in which speakers named pairs of objects. One possibility, which should be investigated in future research, is that this effect is a result of a selective sensitivity of the N2pc to such early attention shifts. But against the background that speech planning strategies are context-dependent, and that several features of the paradigm we employed deviated from earlier studies in order to accommodate EEG recordings, it is possible that the unpredictability of the naming direction, the flickering stimuli, and the requirement to keep the eyes fixated on the center of the screen played a role in eliciting an early effect of second object difficulty. Nevertheless, the fact that this effect was confirmed in two quite different analyses (cluster-based permutation tests and a fractional peak latency measure) demonstrates that there is at least one situation in which speakers attend to the second object at a very early point in time.

In addition to the N2pc, the flickering backgrounds successfully elicited SSVEPs, opening up the possibility of tracking attention allocation to individual objects over time. However, SSVEP amplitude was not reliably influenced by the processing difficulty of the objects. What could explain this absence of evidence for SSVEP modulations, in the context of clear N2pc effects? It is possible that the following differences between the N2pc and SSVEPs contributed to this pattern of results. First, the SSVEP modulations may have been too slow or too variable to push the overall amplitude in a particular direction across trials. Whereas the N2pc can shift between locations every $100 \mathrm{~ms}$ in a certain type of visual search task (Woodman and Luck, 1999), SSVEPs have been estimated to increase in amplitude only after $600-800 \mathrm{~ms}$ relative to the onset of an attention-directing cue (Müller et al., 1998b). Furthermore, the neuronal generators of the components differ: whereas the N2pc has been localized to V4 and the lateral occipital complex (Hopf et al., 2000, 2006; Praamstra and Oostenveld, 2003), associated with intermediate and higher levels of the visual processing pathway, SSVEPs have been localized to primary visual cortex, V1 (Di Russo et al., 2007), associated with very early visual processing. Ultimately, there is a difference in functional role: whereas the N2pc is thought to reflect the focusing of attention on an item (e.g., Luck, 2012), SSVEPs are thought to reflect a gain-control mechanism that enhances the discriminability of attended stimuli (e.g., Müller et al., 1998b). On this account, it appears that the speakers focused attention on each object in turn, but this did not reliably lead to enhanced stimulus processing at that location as a function of whether the object was easy or difficult to process (for a dissociation between detection performance and the N2pc, see Woodman and Luck, 2003a). This could be a result of the stimuli or of the task. Although N2pc shifts have previously been observed in combination with enhanced stimulus processing, as measured by increased early visual responses to the appearance of a probe square, this was in the context of a visual search task which required participants to discriminate between highly similar symbols, namely an upright T among inverted Ts (Luck et al., 1993). Such a task can only be successfully performed when perceptual details of stimuli are thoroughly processed. One can speculate that thorough processing of visual details may be less important when naming line drawings of objects, which are often visually "overspecified" for their identity. For example, attending to a typical chair's seat, its back, or its legs, or getting a rough impression of its contours, would all support the retrieval of the word "chair", with no need for further detailed perceptual discrimination processes.

It is important to note that the nature of the manipulation used in the present study does not allow us to draw conclusions about specific linguistic processes. In the absence of previous N2pc work on language production, and because the EEG is sensitive to confounds in the entire processing stream from visual perception to motor output, this study opted for a previously established and perceptually well-controlled manipulation of object difficulty. But given that the N2pc was affected by this manipulation, and given the early effect of Object 2 difficulty, the results pave the way for future studies to target specific levels of representation, like semantics, syntax and phonology, to find out at which points in time covert attention helps extract which kinds of information from objects in the service of naming them.

In sum, this study found that EEG can be used to track covert attention allocation while speakers name pairs of objects. While SSVEPs were not affected by the processes of interest, the attention shifts indexed by the N2pc reflected the objects' order of mention and 
revealed attention allocation to the second to-be-named object at an early stage. These results help complete the picture of attention allocation during language production.

\section{Acknowledgements}

We thank Svetlana Gerakaki for providing us with the pictures of objects with a canonical orientation and Sophie Kirkels and Annelies van Wijngaarden for assistance with editing the pictures.

\section{Appendix. : Object names}

aap (monkey), ananas (pineapple), appel (apple), auto (car), ballon (balloon), bank (couch), bed (bed), beker (trophy), blik (can), bloem (flower), boerderij (farm), boom (tree), boot (boat), brandweerman (fireman), brievenbus (mailbox), broek (pants), brood (bread), broodrooster (toaster), bureau (desk), bus (bus), clown (clown), dak (roof), doos (box), eekhoorn (squirrel), fiets (bicycle), fles (bottle), fornuis (stove), geit (goat), gieter (watering can), glas (glass), hart (heart), heks (witch), helm (helmet), hond (dog), huis (house), iglo (igloo), jas (jacket), jurk (dress), kaars (candle), kan (pitcher), kangoeroe (kangaroo), kanon (cannon), kast (dresser), kat (cat), katapult (slingshot), kerk (church), kip (chicken), klerenkast (closet), koe (cow), konijn (rabbit), koning (king), kopje (cup), kruiwagen (wheelbarrow), kruk (stool), laars (boot), leeuw (lion), mand (basket), mixer (mixer), molen (windmill), muis (mouse), neus (nose), neushoorn (rhinoceros), olifant (elephant), overhemd (shirt), paard (horse), paddestoel (mushroom), palmboom (palmtree), pan (pot), paraplu (umbrella), pet (hat), piano (piano), pop (doll), priester (priest), revolver (gun), robot (robot), rolstoel (wheelchair), schilderij (picture), schildpad (turtle), schoen (shoe), schommel (swing), schommelstoel (rocking chair), slak (snail), slee (sled), sneeuwpop (snowman), spaghetti (spaghetti), spiegel (mirror), spook (ghost), stoel (chair), stofzuiger (vacuum), stropdas (tie), taart (cake), tafel (table), tank (tank), tent (tent), tijger (tiger), tractor (tractor), trap (stairs), trein (train), trui (sweater), vaas (vase), varken (pig), verpleegster (nurse), vliegtuig (airplane), voet (foot), vos (fox), vrachtwagen (truck), vuur (fire), weegschaal (scales), zadel (saddle), zebra (zebra), zout (salt), zwaan (swan).

\section{References}

Baayen, R.H., Davidson, D.J., Bates, D.M., 2008. Mixed-effects modeling with crossed random effects for subjects and items. J. Mem. Lang. 59 (4), 390-412.

Barr, D.J., Levy, R., Scheepers, C., Tily, H.J., 2013. Random effects structure for confirmatory hypothesis testing: keep it maximal. J. Mem. Lang. 68 (3), 255-278.

Bock, K., Levelt, W.J.M., 1994. Language production: grammatical encoding. In Gernsbacher, M.A. (Ed.), Handbook of Psycholinguistics. Academic Press, London, 945-984.

Costa, A., Caramazza, A., 2002. The production of noun phrases in English and Spanish: implications for the scope of phonological encoding in speech production. J. Mem. Lang. 46 (1), 178-198.

Dell, G.S., 1986. A spreading-activation theory of retrieval in sentence production. Psychol. Rev. 93 (3), 283-321.

Deubel, H., Schneider, W.X., 1996. Saccade target selection and object recognition: evidence for a common attentional mechanism. Vis. Res. 36 (12), 1827-1837.

Di Russo, F., Pitzalis, S., Aprile, T., Spitoni, G., Patria, F., Stella, A., Hillyard, S.A., 2007. Spatiotemporal analysis of the cortical sources of the steady-state visual evoked potential. Hum. Brain Mapp. 28 (4), 323-334.

Eimer, M., 1996. The N2pc component as an indicator of attentional selectivity. Electroencephalogr. Clin. Neurophysiol. 99 (3), 225-234.

Eulitz, C., Hauk, O., Cohen, R., 2000. Electroencephalographic activity over temporal brain areas during phonological encoding in picture naming. Clin. Neurophysiol. 111 (11), 2088-2097.

Fromkin, V.A., 1971. The non-anomalous nature of anomalous utterances. Language, $27-52$.

Ganushchak, L., Christoffels, I., Schiller, N.O., 2011. The use of electroencephalography in language production research: a review. Front. Psychol. 2, 208.

Garrett, M.F., 1980. Levels of processing in sentence production. In: Butterworth, B. (Ed.), Language Production Vol. I. Academic Press, London, 177-220.

Griffin, Z.M., 2001. Gaze durations during speech reflect word selection and phonological encoding. Cognition 82 (1), B1-B14.

Griffin, Z.M., 2004. Why look? Reasons for eye movements related to language production. In: Henderson, J., Ferreira, F. (Eds.), The Interface of Language, Vision, and Action. Taylor and Francis, New York, 213-247.

Griffin, Z.M., Bock, K., 2000. What the eyes say about speaking. Psychol. Sci. 11 (4), 274-279.

Goncharova, I.I., McFarland, D.J., Vaughan, T.M., Wolpaw, J.R., 2003. EMG contamination of EEG: spectral and topographical characteristics. Clin. Neurophysiol. 114, 1580-1593.

Habets, B., Jansma, B.M., Münte, T.F., 2008. Neurophysiological correlates of linearization in language production. BMC Neurosci. 9 (1), 77.

Hickey, C., Di Lollo, V., McDonald, J.J., 2009. Electrophysiological indices of target and distractor processing in visual search. J. Cogn. Neurosci. 21 (4), 760-775.

Hoffman, J.E., Subramaniam, B., 1995. The role of visual attention in saccadic eye movements. Percept. Psychophys. 57 (6), 787-795.

Hopf, J.M., Luck, S.J., Girelli, M., Hagner, T., Mangun, G.R., Scheich, H., Heinze, H.J., 2000. Neural sources of focused attention in visual search. Cereb. Cortex 10 (12), $1233-1241$.

Jescheniak, J.D., Schriefers, H., Garrett, M.F., Friederici, A.D., 2002. Exploring the activation of semantic and phonological codes during speech planning with eventrelated brain potentials. J. Cogn. Neurosci. 14 (6), 951-964.

Kiesel, A., Miller, J., Jolicoeur, P., Brisson, B., 2008. Measurement of ERP latency differences: a comparison of single-participant and jackknife-based scoring methods. Psychophysiology 45, 250-274.

Konopka, A.E., 2012. Planning ahead: how recent experience with structures and words changes the scope of linguistic planning. J. Mem. Lang. 66 (1), 143-162.

Lee, E.K., Brown-Schmidt, S., Watson, D.G., 2013. Ways of looking ahead: hierarchical planning in language production. Cognition 129 (3), 544-562.

Lins, O.G., Picton, T.W., Berg, P., Scherg, M., 1993. Ocular artifacts in EEG and eventrelated potentials I: scalp topography. Brain Topogr. 6 (1), 51-63.

Luck, S.J., 2005. An Introduction to the Event-Related Potential Technique. MIT Press, Cambridge, MA.

Luck, S.J., 2012. Electrophysiological correlates of the focusing of attention within complex visual scenes: N2pc and related ERP components. In: Luck, S.J., Kappenman, E.S. (Eds.), The Oxford Handbook of Event-Related Potential Components. Oxford University Press, New York, 329-360.

Luck, S.J., Fan, S., Hillyard, S.A., 1993. Attention-related modulation of sensory-evoked brain activity in a visual search task. J. Cogn. Neurosci. 5 (2), 188-195.

Luck, S.J., Hillyard, S.A., 1990. Electrophysiological evidence for parallel and serial processing during visual search. Percept. Psychophys. 48 (6), 603-617.

Luck, S.J., Hillyard, S.A., 1994. Spatial filtering during visual search: evidence from human electrophysiology. J. Exp. Psychol.: Hum. Percept. Perform. 20 (5), $1000-1014$.

Luck, S.J., Kappenman, E.S. (Eds.), 2011. The Oxford Handbook of Event-related Potential Components. Oxford University Press, New York.

Malpass, D., Meyer, A.S., 2010. The time course of name retrieval during multiple-object naming: evidence from extrafoveal-on-foveal effects. J. Exp. Psychol.: Learn., Mem., Cogn. 36 (2), 523-537.

Maris, E., 2012. Statistical testing in electrophysiological studies. Psychophysiology 49, $549-565$.

Maris, E., Oostenveld, R., 2007. Nonparametric statistical testing of EEG- and MEGdata. J. Neurosci. Methods 164 (1), 177-190.

Martin, R.C., Crowther, J.E., Knight, M., Tamborello, F.P., Yang, C.L., 2010. Planning in sentence production: evidence for the phrase as a default planning scope. Cognition 116 (2), 177-192.

Mazza, V., Turatto, M., Caramazza, A., 2009. Attention selection, distractor suppression and N2pc. Cortex 45 (7), 879-890.

Meyer, A.S., 2004. The use of eye tracking in studies of sentence generation. In: Henderson, J.M., Ferreira, F. (Eds.), The Interface of Language, Vision, and Action: Eye Movements and the Visual World. Psychology Press, Hove, 191-212.

Meyer, A.S., Ouellet, M., Häcker, C., 2008. Parallel processing of objects in a naming task. J. Exp. Psychol.: Learn., Mem., Cogn. 34 (4), 982.

Meyer, A.S., Sleiderink, A.M., Levelt, W.J., 1998. Viewing and naming objects: eye movements during noun phrase production. Cognition 66 (2), B25-B33.

Meyer, A.S., van der Meulen, F.F., 2000. Phonological priming effects on speech onset latencies and viewing times in object naming. Psychon. Bull. Rev. 7 (2), 314-319.

Miller, J., Patterson, T., Ulrich, R., 1998. Jackknife-based method for measuring LRP onset latency differences. Psychophysiology 35, 99-115.

Morey, R.D., 2008. Confidence intervals from normalized data: a correction to Cousineau (2005). Tutor. Quant. Methods Psychol. 4 (2), 61-64.

Morgan, S.T., Hansen, J.C., Hillyard, S.A., 1996. Selective attention to stimulus location modulates the steady-state visual potential. Proc. Natl. Acad. Sci. USA 93, 4770-4774.

Morgan, J.L., Van Elswijk, G., Meyer, A.S., 2008. Extrafoveal processing of objects in a naming task: evidence from word probe experiments. Psychon. Bull. Rev. 15 (3), $561-565$.

Morgan, J.L., Meyer, A.S., 2005. Processing of extrafoveal objects during multiple-object naming. J. Exp. Psychol.: Learn., Mem., Cogn. 31 (3), 428-442.

Müller, M.M., Hillyard, S.A., 2000. Concurrent recording of steady-state and transient event-related potentials as indices of visual-spatial selective attention. Clin. Neurophysiol. 111, 1544-1552.

Müller, M.M., Picton, T.W., Valdes-Sosa, P., Riera, P., Teder-Sälejärvi, A.W., Hillyard, S.A., 1998a. Effects of spatial selective attention on the steady-state visual evoked potential in the 20-28 Hz range. Cogn. Brain Res. 6, 249-261.

Müller, M.M., Teder-Sälejärvi, A.W., Hillyard, S.A., 1998b. The time course of cortical facilitation during cued shifts of spatial attention. Nat. Neurosci. 1, 631-634.

Ng, K.B., Bradley, A.P., Cunnington, R., 2012. Stimulus specificity of a steady-state visual-evoked potential-based brain-computer interface. J. Neural Eng. 9, 036008.

Oostenveld, R., Fries, P., Maris, E., Schoffelen, J.-M., 2011. FieldTrip: open source 
software for advanced analysis of MEG, EEG, and invasive electrophysiological data. Comput. Intell. Neurosci. 2011, 9.

Oppermann, F., Jescheniak, J.D., Schriefers, H., 2010. Phonological advance planning in sentence production. J. Mem. Lang. 63 (4), 526-540.

Piai, V., Roelofs, A., van der Meij, R., 2012. Event-related potentials and oscillatory brain responses associated with semantic and stroop-like interference effects in overt naming. Brain Res. 1450, 87-101.

Pollatsek, A., Rayner, K., Collins, W.E., 1984. Integrating pictorial information across eye movements. J. Exp. Psychol.: Gen. 113 (3), 426.

Praamstra, P., Kourtis, D., 2010. An early parietal ERP component of the frontoparietal system: EDAN $\neq N 2 p c$. Brain Res. 1317, 203-210.

Praamstra, P., Oostenveld, R., 2003. Attention and movement-related motor cortex activation: a high-density EEG study of spatial stimulus-response compatibility. Cogn. Brain Res. 16 (3), 309-322.

Rayner, K., 1975. The perceptual span and peripheral cues in reading. Cogn. Psychol. 7 (1), 65-81.

Regan, D., 1989. Human Brain Electrophysiology: Evoked Potentials and Evoked Magnetic Fields in Science and Medicine. Elsevier, New York.

Schotter, E.R., Ferreira, V.S., Rayner, K., 2013. Parallel object activation and attentional gating of information: evidence from eye movements in the multiple object naming paradigm. J. Exp. Psychol.: Learn., Mem., Cogn. 39 (2), 365.

Smith, M., Wheeldon, L., 1999. High level processing scope in spoken sentence production. Cognition 73 (3), 205-246.
Smulders, F.T.Y., 2010. Simplifying jackknifing of ERPs and getting more out of it: retrieving estimates of participants' latencies. Psychophysiology 47, 387-392.

Strijkers, K., Holcomb, P.J., Costa, A., 2011. Conscious intention to speak proactively facilitates lexical access during overt object naming. J. Mem. Lang. 65 (4), 345-362.

Ulrich, R., Miller, J., 2001. Using the jackknife-based scoring method for measuring LRP onset effects in factorial designs. Psychophysiology 38 (05), 816-827.

van Turennout, M., Hagoort, P., Brown, C.M., 1998. Brain activitity during speaking: from syntax to phonology in 40 milliseconds. Science 280, 572-574.

Vialatte, F.B., Maurice, M., Dauwels, J., Cichocki, A., 2010. Steady-state visually evoked potentials: focus on essential paradigms and future perspectives. Prog. Neurobiol. 90 (4), 418-438.

Wagner, V., Jescheniak, J.D., Schriefers, H., 2010. On the flexibility of grammatical advance planning during sentence production: effects of cognitive load on multiple lexical access. J. Exp. Psychol.: Learn., Mem., Cogn. 36 (2), 423.

Wilson, G.F., O’Donnell, R.D., 1986. Steady state evoked responses: correlations with human cognition. Psychophysiology 23, 57-61.

Woodman, G.F., Luck, S.J., 1999. Electrophysiological measurement of rapid shifts of attention during visual search. Nature 400, 867-869.

Woodman, G.F., Luck, S.J., 2003a. Dissociations among attention, perception, and awareness during object-substitution masking. Psychol. Sci. 14 (6), 605-611.

Woodman, G.F., Luck, S.J., 2003b. Serial deployment of attention during visual search. J. Exp. Psychol.: Hum. Percept. Perform. 29 (1), 121. 